DIVISION OF THE HUMANITIES AND SOCIAL SCIENCES

CALIFORNIA INSTITUTE OF TECHNOLOGY

PASADENA, CALIFORNIA 91125

A NOTE ON IMPOSSIBILITY THEOREMS

AND SENIORITY RULES

Matthias Hild

California Institute of Technology \&

Jet Propulsion Laboratory

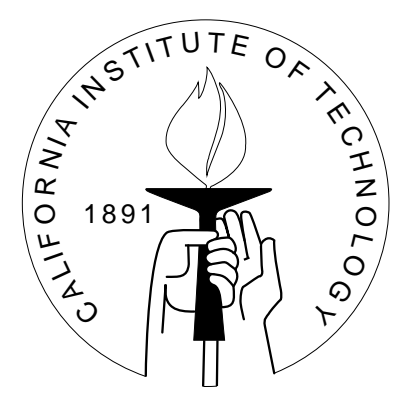

SOCIAL SCIENCE WORKING PAPER 1123

May 2001 


\title{
A Note on Impossibility Theorems and Seniority Rules
}

\author{
Matthias Hild
}

\begin{abstract}
The purpose of a social choice rule is to resolve conflicts among the preferences of a group of individuals. We should therefore require a social choice rules not to remain indecisive between alternatives for which individuals have conflicting preferences. Suppose we also adopt the requirements of a universal domain, strict Pareto optimality and independence of irrelevant alternatives. We then obtain the existence of a dictatorship (for binary choices) already under the weak consistency assumption that the group's choice function must always generate a preference relation that is acyclical over triples of alternatives. By contrast to other theorems, this results holds without any restrictions on the size of the group and without the axiom of positive responsiveness. Under the same consistency condition, we furthermore obtain an axiomatic characterization of seniority rules, also known as lexical dictatorships.
\end{abstract}

JEL classification numbers: D63, D71

Key words: consistency, lexical dictatorship, rationality, seniority, social choice theory 


\title{
A Note on Impossibility Theorems and Seniority Rules
}

\author{
Matthias Hild*
}

In 1785, the Marquis de Condorcet constructed a profile of individual preferences that leads to a cyclical group preference under pairwise majority voting. Suppose the group consists of three individuals 1,2,3 and we have the following rankings of three social alternatives $x, y, z: x \succ_{1} y \succ_{1} z, y \succ_{2} z \succ_{2} x$ and $z \succ_{3} x \succ_{3} y$. If we now hold majority votes in pairwise comparisons of these alternatives, we obtain the following cyclical group preference: $x \succ_{0} y \succ_{0} z \succ_{0} x$. Since this preference relation exhibits a striking form of 'inconsistency', the tradition following Arrow (1951) has focused on exploring which consistency properties, if any, can possibly be satisfied by some appropriate voting mechanism. Apart from the violation of some consistency norm, Condorcet's example raises, however, an additional difficulty for the group's decision making in as far as the group's cyclical preference cannot recommend the choice of any one of the three social alternatives. We may thus criticize pairwise majority voting for remaining indecisive and being unable to resolve the individuals' manifest conflict of interests. In this respect, a large number of voting mechanisms fail their very purpose. When we, for instance, apply de Borda's rule to Condorcet's example, we obtain a group preference that is indifferent between all three alternatives. Though fully rational and consistent, this preference again remains agnostic about how to resolve the individuals' conflict of interests that made the application of some social choice mechanism desirable in the first place. We shall therefore require a social choice rule to be conflict resolving in the sense that it recommends either $x$ or $y$ (but not both) whenever there is some individual who strictly prefers $x$ over $y$ and some individual who strictly prefers $y$ over $x$. Note that this property does not require the societal choice function to be 'resolute' in the sense that it always chooses a unique alternative. Conflict resolution admits the set $\{x, y\}$ as the group choice if all individuals are indifferent between $x$ and $y$ and is thus compatible with the condition of Pareto indifference. We shall apply this resolution condition, firstly, to the derivation of an Arrovian 'impossibility' and, secondly, to the characterization of seniority rules.

Blair, Bordes, Kelly and Suzumura have studied Arrovian impossibilities under the weak consistency condition of base triple-acyclicity. This condition does not impute the existence of some preference relation generating, or 'rationalizing', the group's choice

*Address for correspondence: California Institute of Technology, Mailcode 228-77, Pasadena, CA 91125 (USA). Email: matthias@hild.org. Website: http://www.hild.org. 
function. Suppose the group makes three choices, the first between $x$ and $y$, the second between $y$ and $z$ and the third between $x$ and $z$. If the group chooses $x$ in the first choice and $y$ in the second choice, then base triple-acyclicity requires the group not to choose $z$ in the third choice. Hence, the base relation generated by the group's choice function should be acyclical over all triples of alternatives. In other words, it should not be possible to turn the group into a money-pump by using only three alternatives (although there may exist more sophisticated ways of money-pumping the group by using more than three alternatives). In addition to base triple-acyclicity, Blair, Bordes, Kelly and Suzumura also adopt the conditions of an unrestricted domain, binary independence of irrelevant alternatives and binary weak Pareto optimality. They then obtain the existence of a dictatorship for choices between two alternatives, but only for a group with at least four individuals and only in the presence of positive responsiveness (cf. Blair et al., 1976, Theorem 7 and the remarks in Suzumura, 1983, p. 98). By contrast, we shall derive the existence of a dictatorship without either the axiom of positive responsiveness or a restriction on the number of individuals. Instead, the above resolution condition suffices to derive the existence of a dictatorship for choices between two alternatives from the base triple-acyclicity of the societal choice function, an unrestricted domain, binary independence of irrelevant alternatives and binary strict Pareto optimality.

A second application of the resolution condition concerns a positive characterization of seniority rules under weak consistency assumptions. Seniority rules use an exogenous hierarchy that determines the order in which individuals' preferences are taken into account in an allocation problem. Under this method, the most senior individual first chooses his preferred subset from the feasible set of social alternatives. If this subset contains only one element, then this element is the socially chosen alternative. If the most senior individual chooses a subset with more than one element, then the second most senior individual can choose his preferred alternatives from this subset. The method continues in the same way for all individuals ranked according to their seniority. Many actual allocation procedures are of this type and draw on an exogenous hierarchy in which seniority may be determined by age, waiting time, professional rank etc. (Elster, 1991). Seniority rules stay true to the individuals' preferences but, at the same time, do not require any interpersonal comparisons of welfare on the part of the social decision maker. As a particular example, we have elsewhere examined the allocation of cadaveric donor kidneys on the basis of recipients' waiting time. This allocation method automatically protects disadvantaged patient types who quickly 'bubble up to the top' of the waiting list and thus enjoy priority in the allocation process. Allocation by waiting time thus turns out to preserve what seems to be a set of particularly robust fairness judgments, without requiring the determination of less robust interpersonal trade-offs (Hild, 2001). In this example, a suitable choice of the exogenous hierarchy ensures that the most senior individual is also the morally most deserving individual.

Since seniority rules based on some judiciously chosen hierarchy can thus have morally attractive properties, it is regrettable that these rules have indiscriminately been slandered as 'lexical dictatorships'. Indeed, the most senior individual in a seniority rule fits Arrow's formal definition of a dictator - a term which clearly carries negative connota- 
tions. On a constructive note, Luce and Raiffa (1957) already pointed out that a reinterpretation and a slight technical modification of Arrow's impossibility result provides a positive characterization of the class of seniority rules. To obtain this characterization, we strengthen weak Pareto optimality to strict Pareto optimality and add the condition of Pareto indifference. In the presence of Arrow's strong consistency assumption, the conditions of a universal domain and independence of irrelevant alternatives then deliver the desired result. By how much could we weaken Arrow's consistency assumption and still obtain a positive characterization of seniority rules? The literature does not provide an answer to this question. The above result by Blair, Bordes, Kelly and Suzumura, for instance, is restricted to populations with at least four individuals and thus remains silent on the treatment of the three most junior individuals. Similar restrictions on the size of the group apply to related results (e.g., Theorem 5 by the same authors). This lacuna does not seem to trouble the literature, probably because the existence of an oligarchy or a dictator is predominantly interpreted as an 'impossibility result'. Under this negative interpretation, there is indeed no reason - other than mathematical curiosity - why one should be interested in the conditions under which not just a single dictator but also a hierarchy of dictators exists. Since, in our view, this negative conclusion is premature, we also provide a characterization of seniority rules, both for choices between two alternatives and for choices between an arbitrary number of alternatives. These characterizations use the consistency assumption of base triple-acyclicity and of weak rationalizability, respectively.

\section{Theorem}

Let $X$ be a non-empty set of possible options. Let $\mathbf{O}$ be the set of all orderings on $X$, i.e., of all binary relations on $X$ that are complete and transitive. We write ' $\succ$ ' for the asymmetric part and ' $\sim$ ' for the symmetric part of a binary relation $\succeq$. Let $\mathcal{S}$ be a set of non-empty subset of $X$ such that $\{x, y\} \in \mathcal{S}$ for any $x, y \in X$. C is a choice function if and only if $C: \mathcal{S} \rightarrow \mathcal{S}$ and $C(S) \subseteq S$ for all $S \in \mathcal{S}$. Let $\mathbf{C}$ be the set of all choice functions. A choice function $C$ generates a binary relation $\succeq$ on $X$ exactly when, for all $x, y \in X, x \succeq y$ if only if $x \in C(\{x, y\})$. We write ' $\succeq_{C}$ ' for the relation generated by $C$. This relation is called the 'base relation of' $C$. A binary relation $\succeq$ on $X$ generates a function $C$ if and only if $C(S)=\{x \in S \mid \forall y \in S: x \succeq y\}$ for any $S \in \mathcal{S}$. We write ' $C \succeq$ ' for the choice function generated by $\succeq$. A choice function $C$ is rationalizable if and only if there exists some binary relation $\succeq$ on $X$ that generates $C$. We note that a choice function $C$ is rationalizable if and only if $\succeq_{C}$ generates $C$.

Let $I \in \mathbb{N}^{+}$be a fixed number of individuals. $F$ is a social choice rule if and only if there exists some $\mathcal{O} \subseteq \mathbf{O}^{I}$ such that $F: \mathcal{O} \rightarrow$ C. A social choice rule $F$ is a seniority rule if and only if there is a permutation $\sigma$ of $\{1, \ldots, I\}$ such that, for any $\left\langle\succeq_{i}\right\rangle$ in the domain of $F$ :

(Seniority) $\quad F\left(\left\langle\succeq_{i}\right\rangle\right)=C_{\succeq_{\sigma(I)}} \circ \ldots \circ C_{\succeq_{\sigma(1)}}$ 
We say that a social choice rule $F$ is a seniority rule for binary choices if and only if there is a permutation $\sigma$ of $\{1, \ldots, I\}$ such that, for any $\left\langle\succeq_{i}\right\rangle$ in the domain of $F$ and any $x, y \in X,\left[F\left(\left\langle\succeq_{i}\right\rangle\right)\right](\{x, y\})=\left[C_{\succeq_{\sigma(I)}} \circ \ldots \circ C_{\succeq_{\sigma(1)}}\right](\{x, y\})$. Equivalently, a social choice rule $F$ is a seniority rule for binary choices if and only if there is a permutation $\sigma$ of $\{1, \ldots, I\}$ such that the following holds for any $\left\langle\succeq_{i}\right\rangle$ in the domain of $F$ : (i) $x \succ_{C_{0}} y$ if and only if there is some $1 \leq j \leq I$ such that $x \succ_{\sigma(j)} y$ and $x \sim_{\sigma(k)} y$ for every $1 \leq k<j$ and (ii) $x \sim_{C_{0}} y$ if and only if $x \sim_{\sigma(k)} y$ for every $1 \leq k \leq I$ (where $C_{0}:=F\left(\left\langle\succeq_{i}\right\rangle\right)$ ).

Let $F$ be a social choice rule. We then say that $F$ has a universal domain if and only if its domain is $\mathbf{O}^{I} . F$ is base triple-acyclical if and only if, for any $\left\langle\succeq_{i}\right\rangle$ in the domain of $F$ and for any $x, y, z \in X$, if $C_{0}(\{x, y\})=\{x\}$ and $C_{0}(\{y, z\})=\{y\}$, then $C_{0}(\{x, z\}) \neq\{z\}$ (where $C_{0}:=F\left(\left\langle\succeq_{i}\right\rangle\right)$ ). Equivalently, any choice function $C$ in the domain of $F$ must generate a base relation $\succeq_{C}$ that is triple-acyclical (i.e., for which $x \succ_{C} y$ and $y \succ_{C} z$ implies $z \succ_{C} x$, for any $\left.x, y, z \in X\right)$. F satisfies binary conflict resolution if and only if, for any $\left\langle\succeq_{i}\right\rangle$ in the domain of $F$ and for any $x, y \in X$, if there exist some $1 \leq j, j^{\prime} \leq I$ with $x \succ_{j} y$ and $y \succ_{j^{\prime}} x$, then $C_{0}(\{x, y\}) \neq\{x, y\}$ (where $\left.C_{0}:=F\left(\left\langle\succeq_{i}\right\rangle\right)\right)$. Note that $C_{0}(\{x, y\}) \neq\{x, y\}$ is equivalent to the condition that either $C_{0}(\{x, y\})=\{x\}$ or $C_{0}(\{x, y\})=\{y\}$. Moreover, binary conflict resolution and base triple-acyclicity alone are not sufficient to imply the transitivity (or quasi-transitivity) of the base relation generated by the group's choice function. F satisfies binary independence (of irrelevant alternatives) if and only if, for any $\left\langle\succeq_{i}\right\rangle,\left\langle\succeq_{i}^{\prime}\right\rangle$ in the domain of $F$ and for any $x, y \in X$, if $\succeq_{i}\left|\{x, y\}=\succeq_{i}^{\prime}\right|\{x, y\}$ for all $1 \leq i \leq I$, then $C_{0}(\{x, y\})=C_{0}^{\prime}(\{x, y\})$ (where $C_{0}:=F\left(\left\langle\succeq_{i}\right\rangle\right)$ and $C_{0}^{\prime}:=F\left(\left\langle\succeq_{i}^{\prime}\right\rangle\right)$ ). In view of the confusion that pervades the literature, it is perhaps worth repeating that independence of irrelevant alternatives is an extremely weak property that is even satisfied by de Borda's rule (cf. Plott, 1976 and Bordes/Tideman, 1991). F satisfies binary strict Pareto preference if and only if, for any $\left\langle\succeq_{i}\right\rangle$ in the domain of $F$ and for any $x, y \in X$, if $x \succeq_{i} y$ for all $1 \leq i \leq I$ and $x \succ_{j} y$ for some $1 \leq j \leq I$, then $C_{0}(\{x, y\})=\{x\}$ (where $C_{0}:=F\left(\left\langle\succeq_{i}\right\rangle\right)$ ). F satisfies binary Pareto indifference if and only if, for any $\left\langle\succeq_{i}\right\rangle$ in the domain of $F$ and for any $x, y \in X$, if $x \sim_{i} y$ for all $1 \leq i \leq I$, then $C_{0}(\{x, y\})=\{x, y\}$ (where $C_{0}:=F\left(\left\langle\succeq_{i}\right\rangle\right)$ ). $F$ is rationalizable if and only if any choice function in the range of $F$ is rationalizable, i.e., if and only if any choice function in the range of $F$ is generated by some binary relation on $X$. The concept of rationalizability involves no further assumptions about the properties of the binary relation generating a rationalizable choice function. It follows merely that the base relation generated by a rationalizable choice function must be acyclical. Finally, we say that $j(1 \leq j \leq I)$ is a (binary) dictator for $F$ if and only if, for every $\left\langle\succeq_{i}\right\rangle$ in the domain of $F$ and any $x, y \in X$, we have $C_{0}(\{x, y\})=\{x\}$ whenever $x \succ_{j} y$ (where $\left.C_{0}:=F\left(\left\langle\succeq_{i}\right\rangle\right)\right)$.

Theorem 1 (Binary Dictatorship) Assume that $X$ has at least three elements. If $F$ is a social choice rule that has a universal domain and which satisfies base tripleacyclicity, binary conflict resolution, binary independence, binary strict Pareto optimality, then there exists a binary dictator for $F$.

Theorem 2 (Binary Seniority Rules) Assume that $X$ has at least three elements and that $F$ is a social choice rule with a universal domain. Then $F$ is a seniority rule for 
binary choices if and only if F satisfies base triple-acyclicity, binary conflict resolution, binary independence, binary Pareto indifference and binary strict Pareto optimality.

Corollary 3 (Seniority Rules) Assume that $X$ has at least three elements and that $F$ is a social choice rule with a universal domain. Then $F$ is a seniority rule if and only if $F$ is rationalizable and satisfies binary conflict resolution, binary independence, binary Pareto indifference and binary strict Pareto optimality.

\section{Proofs}

We draw on results by Gibbard (1969), Sen (1970) and Mas-Colell/Sonnenschein (1972) who derive the existence not of a dictator, but of an oligarchy under the assumption that the societal choice function always generates a quasi-transitive base relation. Let $V^{*}:=\{1, \ldots, I\}$. Let $\mathcal{V}$ be the set of all subsets of $V^{*}$ and let $\mathcal{V}^{+}$be the set of all non-empty subsets of $V^{*}$. For any $V \in \mathcal{V}^{+}$and any $W \in \mathcal{V}$ with $V \cap W=\varnothing$, we say that $V$ is decisive for $F$ on $\langle x, y\rangle \in X^{2} \operatorname{modulo} W$ if and only if, for every $\left\langle\succeq_{i}\right\rangle$ in the domain of $F$, we have $\left[F\left(\left\langle\succeq_{i}\right\rangle\right)\right](\{x, y\})=\{x\}$ whenever $x \succ_{j} y$ for all $j \in V$ and $x \sim_{k} y$ for all $k \in W$. For any $V \in \mathcal{V}^{+}$and any $W \in \mathcal{V}$ with $V \cap W=\varnothing$, we say that $V$ is almost decisive for $F$ on $\langle x, y\rangle \in X^{2}$ modulo $W$ if and only if, for every $\left\langle\succeq_{i}\right\rangle$ in the domain of $F$, we have $\left[F\left(\left\langle\succeq_{i}\right\rangle\right)\right](\{x, y\})=\{x\}$ whenever $x \succ_{j} y$ for all $j \in V, y \succ_{j^{\prime}} x$ for all $j^{\prime} \in V^{*}-V-W$ and $x \sim_{k} y$ for all $k \in W$. For any $V \in \mathcal{V}^{+}$and any $W \in \mathcal{V}$ with $V \cap W=\varnothing$, we say that $V$ is globally decisive for $F$ modulo $W$ if and only if $V$ is decisive for $F$ on any $\langle x, y\rangle \in X^{2}$ modulo $W$. For any $1 \leq j \leq I$ and $W \in \mathcal{V}$ with $j \notin W, j$ is a (binary) dictator for $F$ modulo $W$ if and only if $\{j\}$ is globally decisive for $F$ modulo $W$.

Lemma 4 Assume that $X$ has at least three elements and that $F$ is a social choice rule that has a universal domain and which satisfies base triple-acyclicity, binary conflict resolution, binary independence and binary strict Pareto optimality. Then for any $W \subset$ $V^{*}$, there exists some $j \in V^{*}-W$ such that $j$ is a binary dictator for $F$ modulo $W$.

Proof of lemma: Suppose that $W \subset V^{*}$. Step 1: We establish that any coalition $V \in \mathcal{V}^{+}$with $V \cap W=\varnothing$ is globally decisive for $F$ modulo $W$ if it is almost decisive for $F$ on some pair $\langle x, y\rangle \in X^{2}$ modulo $W$ and if $x \neq y$. Assume that $V \in \mathcal{V}^{+}$with $V \cap W=\varnothing$ is almost decisive for $F$ on some pair $\langle x, y\rangle \in X^{2}$ modulo $W$ and that $x \neq y$. Suppose that $\left\langle x^{*}, y^{*}\right\rangle \in X^{2}$. We claim that $V$ is decisive for $F$ on $\left\langle x^{*}, y^{*}\right\rangle$ modulo $W$. If $x^{*}=y^{*}$, then the claim is trivial. Suppose therefore that $x^{*} \neq y^{*}$.

Case 1: The set $\{x, y\} \cap\left\{x^{*}, y^{*}\right\}$ is empty. Hence, $x, y, x^{*}$ and $y^{*}$ are four distinct elements of $X$. Then there exists some $\left\langle\succeq_{i}\right\rangle \in \mathbf{O}^{I}$ such that the restriction of $\succeq_{i}$ to $\left\{x, y, x^{*}, y^{*}\right\}$ satisfies the following conditions: $x^{*} \succ_{i} x \succ_{i} y \succ_{i} y^{*}$ for any $i \in V, y \succ_{i} x^{*} \succ_{i} x$ and $y \succ_{i} y^{*} \succ_{i} x$ for any $i \in V^{*}-V-W$, and $x \sim_{i} y \sim_{i} x^{*} \sim_{i} y^{*}$ for any $i \in W$. By the assumption of a universal domain, $C_{0}:=F\left(\left\langle\succeq_{i}\right\rangle\right)$ is well-defined. Let $\succeq_{0}$ be the binary relation generated by $C_{0}$. By binary strict Pareto optimality, $x^{*} \succ_{0} x$ and $y \succ_{0} y^{*}$. Since $V$ is almost decisive for $F$ on $\langle x, y\rangle$ modulo $W$, we have $x \succ_{0} y$. By base triple-acyclicity, 
we must have $x^{*} \succeq_{0} y$. By binary conflict resolution, we obtain $x^{*} \chi_{0} y$ whence $x^{*} \succ_{0} y$. Applying base triple-acyclicity once more, we obtain $x^{*} \succeq_{0} y^{*}$. If $y^{*} \succ_{i} x^{*}$ for some $i \in V^{*}-V-W$, then binary conflict resolution implies $x^{*} \succ_{0} y^{*}$. If $x^{*} \succeq_{i} y^{*}$ for all $i \in V^{*}-V-W$, then by binary strict Pareto optimality also yields $x^{*} \succ_{0} y^{*}$. In any case, we have $x^{*} \succ_{0} y^{*}$. Binary independence then implies that $V$ is decisive for $F$ on $\left\langle x^{*}, y^{*}\right\rangle$ modulo $W$.

Case 2: The set $\{x, y\} \cap\left\{x^{*}, y^{*}\right\}$ contains exactly one element. There are four possible cases: $x=x^{*}, x=y^{*}, y=x^{*}$ or $y=y^{*}$. We only consider the sub-case where $x=x^{*}$. We then must have $y \neq y^{*}$. Thus, $x, y$ and $y^{*}$ must be three distinct elements of $X$. Then there exists some $\left\langle\succeq_{i}\right\rangle \in \mathbf{O}^{I}$ such that the restriction of $\succeq_{i}$ to $\left\{x, y, y^{*}\right\}$ satisfies the following conditions: $x \succ_{i} y \succ_{i} y^{*}$ for any $i \in V, y \succ_{i} x$ and $y \succ_{i} y^{*}$ for any $i \in V^{*}-V-W$ and $x \sim_{i} y \sim_{i} y^{*}$ for any $i \in W$. By the assumption of a universal domain, $C_{0}:=F\left(\left\langle\succeq_{i}\right\rangle\right)$ is well-defined. Let $\succeq_{0}$ be the binary relation generated by $C_{0}$. By binary strict Pareto optimality, $y \succ_{0} y^{*}$. Since $V$ is almost decisive for $F$ on $\langle x, y\rangle$ modulo $W$, we have $x \succ_{0} y$. Base triple-acyclicity implies $x \succeq_{0} y^{*}$. If $y^{*} \succ_{i} x$ for some $i \in V^{*}-V-W$, then binary conflict resolution implies $x \succ_{0} y^{*}$. Otherwise, binary strict Pareto optimality yields $x \succ_{0} y^{*}$. In any case, binary independence implies that $V$ is decisive for $F$ on $\left\langle x, y^{*}\right\rangle=\left\langle x^{*}, y^{*}\right\rangle$ modulo $W$.

Case 3: The set $\{x, y\} \cap\left\{x^{*}, y^{*}\right\}$ contains exactly two elements. Case 3.1: $x^{*}=y$ and $y^{*}=x$. Since $X$ has at least three elements, there exists some $z \in X-\{x, y\}$. By Case 2, since $V$ is almost decisive for $F$ on $\langle x, y\rangle$ modulo $W, V$ is decisive (and, hence, almost decisive) for $F$ on $\langle z, y\rangle$ modulo $W$. Again by Case 2, $V$ must therefore be decisive (and, hence, almost decisive) for $F$ on $\langle z, x\rangle$ modulo $W$. Again by Case $2, V$ is thus decisive for $F$ on $\langle y, x\rangle=\left\langle x^{*}, y^{*}\right\rangle$ modulo $W$. Case 3.2: $x^{*}=x$ and $y^{*}=y$. Since $V$ is almost decisive for $F$ on $\langle x, y\rangle$ modulo $W$, the result of the previous case 3.1 implies that $V$ is decisive (and, hence, almost decisive) for $F$ on $\langle y, x\rangle$ modulo $W$. The same result then also implies that $V$ is decisive for $F$ on $\langle x, y\rangle$ modulo $W$.

Step 2: We now show that there exists a binary dictator for $F$ modulo $W$. Let $\mathcal{D}$ be the set of all coalitions $V \in \mathcal{V}^{+}$with $V \cap W=\varnothing$ that are globally decisive for $F$ modulo $W$. By binary strict Pareto optimality, the set $V^{*}-W$ is globally decisive for $F$ modulo $W$ and, hence, $\mathcal{D}$ is non-empty. Hence, there exists some 'smallest' coalition $V_{0} \in \mathcal{D}$ such that $\left(^{*}\right)$ any proper subset of $V_{0}$ is not in $\mathcal{D}$. Suppose $j \in V_{0}$. We now show that $\{j\}$ is globally decisive for $F$ modulo $W$. By assumption, there exists three different alternatives $x, y, z \in X$. Hence, there exists some $\left\langle\succeq_{i}\right\rangle \in \mathbf{O}^{I}$ such that the restriction of $\succeq_{i}$ to $\{x, y, z\}$ satisfies the following conditions: $y \succ_{j} z \succ_{j} x, x \succ_{i} y \succ_{i} z$ for any $i \in V_{0}-\{j\}, z \succ_{i} x \succ_{i} y$ for any $i \in V^{*}-V_{0}-W$, and $x \sim_{i} y \sim_{i} z$ for any $i \in W$. Since, by assumption, $V_{0}$ is globally decisive for $F$ modulo $W$, we have $y \succ_{0} z$. If we had $x \succ_{0} z$, then $V_{0}-\{j\}$ would be almost decisive for $F$ on $\langle x, z\rangle$ modulo $W$ (by binary independence). By Step 1, $V_{0}-\{j\}$ would then also be globally decisive, in contradiction with $(*)$. Hence, we must have $z \succeq_{0} x$ and, by binary conflict resolution, $z \succ_{0} x$. By base triple-acyclicity, we obtain $y \succeq_{0} x$ and, again by binary conflict resolution, $y \succ_{0} x$. By binary independence, we conclude that $j$ is almost decisive for $F$ on $\langle y, x\rangle$ modulo $W$. By Step $1, j$ is then a binary dictator for $F$ modulo $W$. (Incidentally, this means that $V_{0}=\{j\}$.) 
Proof of Theorem 1: According to our earlier definition, it follows that $j$ is a binary dictator for $F$ if and only if $j$ is a binary dictator for $F$ modulo $\varnothing$. The lemma then establishes the claim.

Proof of Theorem 2: It is easy to show that a seniority rule for binary choices satisfies the properties listed in the theorem. We therefore suppose that $F$ satisfies these properties and show that $F$ is a seniority rule for binary choices. By our lemma, there exists some $1 \leq j_{1} \leq I$ such that $j_{1}$ is a binary dictator for $F$. Define $\sigma(1):=j_{1}$. By the same lemma, there exists a binary dictator $j_{2}$ for $F$ modulo $\left\{j_{1}\right\}$. Define $\sigma(2):=j_{2}$ and continue analogously until $\sigma$ is defined for any $1 \leq i \leq I$. Suppose that $\left\langle\succeq_{i}\right\rangle \in \mathbf{O}^{I}$ and $x, y \in X$. Let $\succeq_{0}$ be the binary relation generated by $F\left(\left\langle\succeq_{i}\right\rangle\right)$. We have to show that (**) $x \succ_{0} y$ if and only if there is some $1 \leq j \leq I$ such that $x \succ_{\sigma(j)} y$ and $x \sim_{\sigma(k)} y$ for every $1 \leq k<j$, and $x \sim_{0} y$ if and only if $x \sim_{\sigma(k)} y$ for every $1 \leq k \leq I$. If $x \sim_{i} y$ for all $1 \leq i \leq I$, then $x \sim_{0} y$ (by binary Pareto indifference) and (**) is trivially satisfied. Suppose therefore that there is some $1 \leq i \leq I$ with $x \chi_{i} y$. Let $k(1 \leq k \leq I)$ be the smallest number such that $x \chi_{\sigma(k)} y$. Without loss in generality, we can assume that $x \succ_{\sigma(k)} y$. Let $W:=\{\sigma(l) \mid 1 \leq l<k\}$ and note that $x \sim_{i} y$ for all $i \in W$. By definition of $\sigma$, we know that $\sigma(k)$ is a binary dictator for $F$ modulo $W$. Hence, $\left[F\left(\left\langle\succeq_{i}\right\rangle\right)\right](\{x, y\})=\{x\}$. This establishes claim $(* *)$.

Proof of Corollary 3: It is again easy to show that a seniority rule satisfies the properties listed in the corollary. We therefore suppose that $F$ satisfies these properties and show that $F$ is a seniority rule. We note that a rationalizable choice function always generates a base relation that is triple-acyclical. Hence, the previous theorem implies

that $F$ is a seniority rule for binary choices. Since $F$ is rationalizable, it must also be a seniority rule for arbitrary choice sets.

\section{References}

[1] K. Arrow. Social Choice and Individual Values. Wiley, New York, 1951.

[2] D.H. Blair, G. Gordes, J.S. Kelly, and K. Suzumura. Impossibility theorems without collective rationality. Journal of Economic Theory, 13:361-379, 1976.

[3] G. Bordes and N. Tideman. Independence of irrelevant alternatives in the theory of voting. Theory and Decision, 30:163-186, 1991.

[4] J. Elster. Local justice and interpersonal comparisons. In J. Elster and J.E. Roemer, editors, Interpersonal Comparisons of Well-Being, pages 200-254. CUP, Cambridge, 1991.

[5] A. Gibbard. Social choice and the Arrow conditions. Unpublished manuscript, 1969.

[6] M. Hild. Fair kidney allocation based on waiting time. California Institute of Technology, Social Science Working Paper, 1117; also forthcoming in Analyse und Kritik, 2001. Available at www.hild.org.

[7] R.D. Luce and H. Raiffa. Games and Decisions. John Wiley, 1957.

[8] A. Mas-Colell and H. Sonnenschein. General possibility theorems for group decisions. Review of Economic Studies, 39:185-92, 1972. 
[9] C. Plott. Axiomatic social choice theory: An overview and interpretation. American Journal of Political Science, 20:511-596, 1976.

[10] A. Sen. Collective Choice and Social Welfare. Holden-Day, San Fransisco, 1970.

[11] K. Suzumura. Rational Choice, Collective Decisions, and Social Welfare. CUP, Cambridge, 1983. 